\title{
Diagramming Objections to Independent Premises
}

\section{Cathal Woods}

Dept. of Philosophy

Virginia Wesleyan College

Norfolk, VA 23502

USA

cwoods@vwc.edu

\begin{abstract}
Arguments involving what are called "independent" or "convergent" premises are typically diagrammed in informal logic texts by using multiple arrows, one between each premise and the conclusion. Such diagrams can be confused with other diagrams, can obscure an important ambiguity and make diagramming objections to the reasoning difficult. In response, I suggest that a single arrow should be used for such arguments and a split-tailed arrow when the structure of an independent-premise argument is indeterminate. I then discuss methods for diagramming objections, support for objections and rebuttals to objections.
\end{abstract} Resumé:

Keywords: critical reasoning, critical thinking, dependent, diagram, independent, informal logic, objection, premise, rebuttal, split-tail arrow.

\section{Introduction}

I agree with authors such as Toulmin (1958), Johnson \& Blair (1994), and Walton (2006) in thinking of critical reasoning as a flexible tool of educated citizens. I also concur that we want students to be able to tackle long and structurally complex arguments, such as occur between more than one person or appear in more thoughtful editorials, not to mention in the work of professional arguers. These arguments also often contain objections, as well as support for and rebuttals to those objections. The ability to "follow the dialectic"- to keep track of claims,

(C) Cathal Woods. Informal Logic, Vol. 31, No. 2 (2011), pp. $139-151$ 
reasons, objections and rebuttals that might stretch over minutes of speech or pages of text - is rightly encouraged.

Diagramming or mapping has greater potential than standard form does for representing longer, more complex arguments, including objections, support for objections and rebuttals, ${ }^{1}$ since it uses space, directional arrows and other symbols in order to convey more about the relationship between propositions. Numbers can take the place of propositions, allowing for greater space on the page and increased focus on the relationships between propositions. The increased clarity of the structure of the argument is then an aid in the process of its evaluation, whether in eristic or philosophical contexts.

In section 2 , I point out various difficulties with a standard way of diagramming what are typically called "independent" or "convergent" premises, and shall furthermore argue that the basic diagram makes the inclusion of objections difficult. This difficulty is because multiple arrows are used in such arguments, one for each premise, whereas diagramming objections requires that all of our simple diagrams involve a single arrow for each (attempted) inference.

The most challenging case for rendering (initial) arguments with a single arrow is the kind of multiple-premise argument - which has been thought to be, or be a form of, independent-premise argument-in which the speaker simply throws out one reason $^{2}$ on top of another without regard for structure; these might seem to require multiple arrows. Instead I suggest that we use a split-tailed arrow that represents both the single attempt to convince and the possibility that any one premise (or any subset, if there are more than two), or both together, are sufficient.

In section 3, I survey methods for diagramming objections (and support for objections and rebuttals to objection) generally. (That is, including, but not limited to, independentpremise arguments.)

1 There are additional tasks that diagramming might take on, such as representing conditional arguments and reductios. In this article I limit myself to objections, support for objections and rebuttals, and even more narrowly to a particular problem in diagramming them.

${ }^{2}$ A reason can be expressed in more than one premise. But the distinction between 'reason' and 'premise' does not impact the content of this paper. 


\section{Independent/Convergent Premises}

In a diagram, the propositions or (often) numbers standing for the propositions are used along with arrows and various symbols or words indicating the relationships between propositions. $\mathrm{Pa}-$ rentheses, braces or underlining might also be used to clarify groups of numbers and symbols.

Textbooks that cover diagramming will usually cover arguments with a single premise and single conclusion, arguments with multiple premises and a single conclusion, and extended (or: compound) arguments. Among multiple-premise arguments, a distinction is made between arguments with dependent or linked premises and independent or convergent premises.

Instantiations of simple valid forms, such as modus ponens (a.k.a. Affirming the Antecedent), provide paradigmatic examples of dependent or linked premises. For example (Argument 1):

(1) If Jack is in Baghdad, he is in Iraq.

(2) Jack is in Baghdad.

(3) Jack is in Iraq.

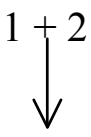

This diagram uses the conventions of numbers standing for the propositions listed on the left, a plus-sign between premises indicating the dependence or linkage of the premises, and a single arrow between premises and conclusion indicating the premises' support for the conclusion.

There is no paradigm of arguments with independent premises because the notion is variously understood. The typical diagram, however, is always the same, even as its interpretation is varied. The following argument (Argument 2) is offered without comment:

(1) Snakes on a Plane has a great concept.

(2) Snakes on a Plane stars Samuel L. Jackson.

(3) Snakes on a Plane is a good movie.

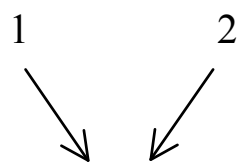


Here, no plus sign or other indication of a relation between (1) and (2) is present. Two arrows are used, one between each premise and the conclusion. ${ }^{3}$

What is the meaning of the arrow in these diagrams? One possibility is that it means "is (intended to) justify belief of", 4 since speakers by default give an argument (whether it consists of one reason or more) that they hope will be found convincing, and the arrow takes the place of conclusionindicator words such as "so" or "therefore".

If the arrow means "justifies", however, the diagram for the independent-premise Argument 2 will be interpreted as showing two premises, each of which is sufficient to justify the conclusion. This would imply that we have two separate arguments that point towards the same conclusion. And indeed, a single passage containing two reasons explicitly said to each be sufficient could be diagrammed this way (or could be diagrammed separately, writing the conclusion number out twice). However, a speaker might make clear that the support from two (or more) independent premises together provides sufficient justification for the conclusion to follow (or, be supposed to follow) with a high degree of confidence, each premise lending only moderate support to the conclusion. The meaning of each arrow might instead be "supports" (in the sense of "lends some, non-sufficient, support to") rather than "justifies". 5 However, we cannot assign the arrow the meaning of non-sufficient support in every case because arguments with a single arrow (whether because there is a single premise or a single set of dependent premises) are presented as being (or intended to be) sufficient, and the premises (are supposed to) justify the conclusion.

In response, we might suggest a meaning that is indeterminate as to sufficiency: "supports and is/are possibly sufficient for". But a two-arrow diagram with this meaning is the same as a diagram in which there are two sufficient reasons for a

3 To my knowledge, this way of diagramming independentpremise arguments is found in all treatments of diagramming, and is built into Araucaria and Austhink.

${ }^{4}$ For simplicity I shall often abbreviate to "justifies". In fact, since speakers can lie or be deceptive, the basic meaning should more cautiously be taken only to be "is intended to convince the audience of".

${ }^{5}$ I disagree with Yanal's (1991) contention that there are two (or more) arguments in an argument with independent premises ( $\mathrm{p}$. 139), though perhaps he understands the arrow here to mean "supports" rather than "justifies" or "therefore". To my mind, a better default position is to say that we do not know what the structure of the argument is, as will be discussed momentarily. 
conclusion; we cannot tell by looking at the diagram whether the two are indeed separate or to be taken together. In response, we might dispense with multiple arrows and say that if it is clear that the support from multiple reasons is to be added, a single arrow can be used, along with some spatial device or symbol other than a plus sign (which is already in service for dependentpremise arguments) between or around the numbers representing the premises. ${ }^{6}$

There is thus no need to use to multiple arrows for arguments involving added support. But there are, in addition, arguments involving independent reasons without any indication of what degree of support each gives. In my experience, thismultiple reasons without clarifying verbiage - is the most common form of argumentation in public forums such as newspaper editorials, blogs and television talking heads. It is unclear whether each premise is a separate argument or whether only the two added together are sufficient. To diagram such arguments as either multiple arguments or added support artificially resolves this ambiguity. Perhaps such cases should be diagrammed using multiple arrows, (perhaps) since we will have to examine each reason and the support (whether sufficient or not) it gives to the conclusion in turn, but the proposal to use a split-tailed arrow, which will be introduced in a moment, requires this while preserving the initial ambiguity

A stronger reason to avoid multiple arrows in a diagram (of any type of argument) is that multiple arrows make diagramming passages with objections difficult. Consider the diagram below, for an argument (Argument 3) that (1) we (the speaker and audience) should get a dog because (2) it will protect the house and (3) it will be fun for the kids. Imagine that this argument is diagrammed with two arrows. (It does not matter whether speaker intends added support or two sufficient reasons.)

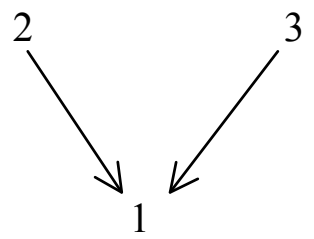

\footnotetext{
${ }^{6}$ Any number of symbolic devices could be employed to mark the difference. One option is underlining or a bar beneath the two, with a single arrow. Another is use of parentheses. One might stack the premise numbers vertically, conveying the idea of weight. From the audience at the AAPT, Bill Melanson suggested using a comma.
} 
Now imagine an objection to the effect that, even granting the truth of both, (1) does not securely follow (even if the support from each is added). For example, one might object that there's more to getting a dog than these two factors and some of the additional concerns go against the idea. In the few works that have attempted to give a system of diagramming for objections, the challenge will be represented by a number standing for a proposition and an arrow of some sort pointing away from this number. At what would we point this challenging arrow?

One thought might be that we can simply draw the objection-arrow pointing at (1), the conclusion. This is, indeed, how some existing attempts to incorporate objections proceed. However, there are various forms of objection and any single method for diagramming objections will necessarily obscure these differences. We might hope to distinguish objections to the truth of a premise from those to the strength of the inference. Kelley (1988), Thomas (1986) and Austhink allow this difference to be diagrammed. Moreover, pointing directly at the conclusion might be reserved for counter-arguments, that is, for arguments that the conclusion is false (that its negation is true), as distinct from objections aimed only at the truth of a premise or at the reasoning from premises to conclusion of the given argument. Consider the difference between "There's more to getting a dog than protection and fun." and "We can't afford a dog.". It is true that all counter-arguments imply a fault with the original argument, but not all objections assert that the conclusion is false; some say only that it cannot securely be accepted.

How might we diagram these two types of objection? An objection to the truth of a premise is easily handled-it would point at the premise in question. If the objection is to the inference from premises to conclusion, we might point it at an arrow which represents the inference. But there are two arrows in this diagram and we can't neatly draw it against both of them. Perhaps multiple (objecting) arrows could be drawn, one pointing at each of the existing arrows, but, even putting aside the fact that the diagram will quickly be made dense and difficult, it's hard to see how this would represent an objection to the claim that the two together are sufficient to justify the conclusion. Arguments with more than two arrows will present even greater difficulty in this regard.

Another alternative, employed by Kelley (1988, p. 155) and hinted at by Epstein (2002, p. 157), is to demand a connecting premise, the purpose of which is to express the reasoning. Argument (3), which provided reasons for getting a dog, might be modified as follows: 


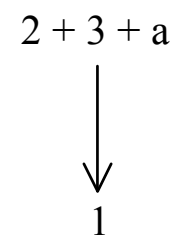

In this diagram, the proposition (a) is the additional, connecting, premise, ${ }^{7}$ which clarifies that (2) and (3) go together (and with (a)) in order to justify the conclusion. For example, (a) might be "If a dog will protect the house and be fun for the kids, we should get one.". An objection to the inference now becomes an objection to (a), and the objection arrow can be drawn pointed at single part of the diagram, namely (a).

This strategy requires either that the speaker can be queried or a decision be made on the part of the audience as to what the speaker intended. But the former is often not possible and the latter might be wrong and exclude an option that we should look at when we come to evaluate the argument. The possibility of the different types of independent-premise arguments and the ambiguity in unstructured arguments mean that we can't confidently add a connecting premise, or at least, not prior to a significant step in evaluation. I take it, however, that in such cases some analysis of the argument's structure comes prior to the kind of thinking that would resolve the ambiguity in unstructured arguments ${ }^{8}$ and is based on what if anything the speaker

7 This diagram uses Kelley's convention of giving letters to added premises (but not his use of underlining). Epstein (2002) has his examples (on pp. 360-1) all begin with multiple arrows pointing at the same conclusion, but then resolves them, by the addition of a connecting premise, into diagrams with a single arrow pointing at the conclusion. (Notice that this might involve a confusion in the meaning of the arrow. He initially says that the arrow means "is a reason to believe the other claim" (p. 359) but declares on p. 362 that the arrow is to be understood as "therefore". Subsequent text, however, appears to show that it is being used as "supports"-in the sense of "provides some support"—as was originally stated on p. 358 ("supports") and p. 359.)

${ }^{8}$ An initial analysis does not preclude revision of a diagram in the course of evaluation. Analysis and evaluation impact one another as the audience attempts to do justice to the intention of the speaker while also finding the strongest interpretation of the argument in order to have the best chance of learning from it. 
says concerning the structure, on contextual clues and on familiarity with basic reasoning. ${ }^{9}$

However, as mentioned above, the problem of diagramming objections arises as soon as there is more than one arrow. Only when there two sufficient arguments are clearly intended can we safely use two arrows. Cases of added support can be diagrammed with a single arrow, as described above, but in cases of a structure-less pile of reasons we have to allow for both possibilities.

In order to resolve these issues-both the difficulty of diagramming objections to arguments with independent premises (whether the structure is clearly one of added support or is indeterminate) and the basic difficulty of diagramming unstructured arguments, I suggest that we use a split-tailed arrow for unstructured arguments, as follows:

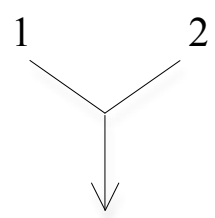

3

The split-tailed arrow is a hybrid of one arrow and two arrows. It represents the audience's ignorance as to whether either or each of the reasons offered is individually sufficient to justify belief in the conclusion or only both together. That is, by its use we show that we do not know whether there are two arguments for the same conclusion (which can be thought of as the shaft unzipping), or added support from the different reasons (or even a dependence relationship which we have failed to recognize). The split-tailed arrow is the default option and the equivalent of the practice in standard form of simply writing the premises in a single vertical list. The combination of multiple tails and a single shaft captures the ambiguity of unstructured arguments; the single shaft is important with respect to diagramming objections to the reasoning in an argument. We turn now to the issue of diagramming objections.

\footnotetext{
${ }^{9}$ See Snoeck Henkemans (1992) for a book-length treatment of "pragmatic", "dialogical" and "dialectical" clues.
} 


\section{Diagramming Objections}

In this section, I present some ways of diagramming objections, support for objections and rebuttals to objections. These methods apply to all of the basic diagrams mentioned above, given that they all involve a single arrow, where the split-tailed arrow is a kind of single arrow.

In textbooks, there is some consensus on using arrows of a different type from those indicating justification/support. Epstein (2002), Kelley (1988), and Moore \& Parker (2001) use an arrow with horizontal marks through them (which I'll call "hasharrows") to represent an objection; Johnson \& Blair (1994) and Thomas (1986) use dashed arrows. (Yoshimi (2004) suggests arrows for objections and lines with a t-bar head for supports.) Further, any premises that support an objection are uniformly given regular (un-hashed, solid) arrows, while rebuttals to those get hash-arrows or dashed arrows.

To repeat a little of section 2, we must first allow for the difference between objections to the truth of a premise, to the strength of the support, and arguments which maintain that the conclusion is false. We might implement the following convention: arrows representing objections to the truth of a premise should point at the number representing the proposition, while challenges to the inference should point at the arrow. When the split-tailed arrow is used (in the case of indeterminate structure) the arrow representing the objection can point at the main shaft, or any of the tails. In the following diagram, (4) is an objection to the truth of (2), (5) is a challenge to the strength of the support, and (6) is a direct argument for the falsity of (1).

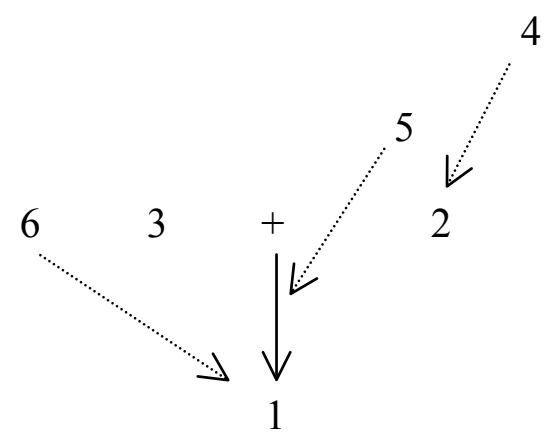

An alternative method is to use a single type of arrow but vary its direction - upward as well as downward - in order to represent objections. (Thomas (1986) has arrows representing a challenge to the reasoning point side-ways at the arrow.) Using this convention, the diagram just above becomes 


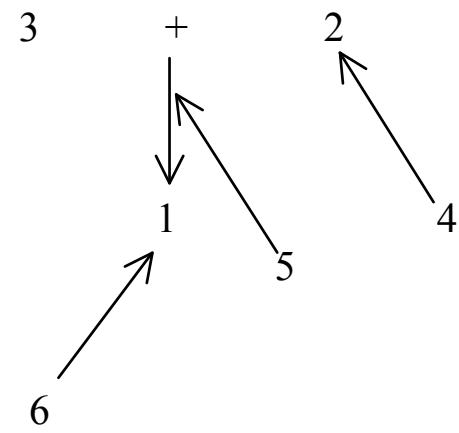

How we should diagram support for, and rebuttals to, objections? Epstein, who uses downward arrows of different types, suggests that regular arrows should be used for supports to objections, just as they are used for supporting premises in the original argument. For example, in the next diagram the arrow between (7) and (6) is the same as that from (3) and (2) to (1). (8), an objection to (6), is connected to it with a dashed arrow:

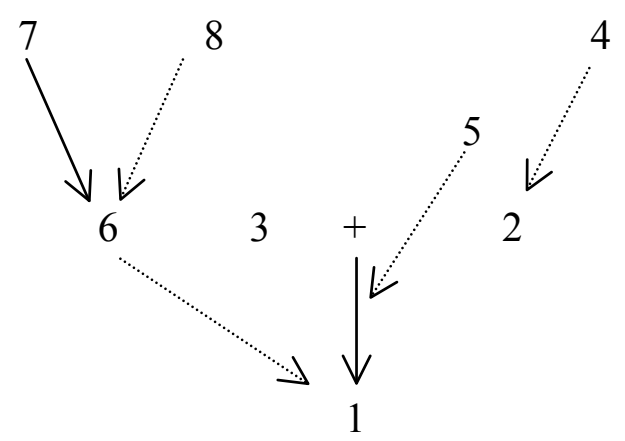

But note two potential confusions. The first is that the arrow from (7) to (6) is the same type as the arrow from (3) and (2) to (1), even though (7) supports (6) in its challenge to (1). (As Austhink cautions, "Note that the reason here helps the objection, not the main contention. It provides evidence that the objection is a good one." See:

http://austhink.com/reason/tutorials/Tutorial_4/2_reason_objecti on/reason_objection.htm ${ }^{10}$ ) Secondly, objections to objections

${ }^{10}$ The Rationale software allows for use of different colors and labels the connecting lines with the words "supports", "opposes" and "rebuts", as appropriate. I thank an anonymous reviewer for bringing this to my attention. 
look identical to objections, even though the former are indirectly supporting the main conclusion. For example, (8) to (6) gets the same type of arrow as between (6) and (1), even though (8) is indirectly supporting (1).

As an alternative, we might again use the direction of the arrow rather than different types of arrow, making all propositions supporting the conclusion (whether directly or indirectly) point downward, while any challenging it (whether directly or indirectly) point upward. Consider the following:

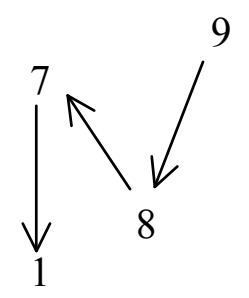

In this diagram, (9) is pushing down against (8)'s attack on (7). It is similar in its direction to the arrow from (7) to (1).

In this scheme, the role of each arrow in the overall argument is immediately clear, but determining whether an arrow supports or challenges what it points at is now more complicated, as it depends on the direction of the other arrow involved. For example, an objection to an objection will point downwards, in the same direction as the original supporting premises. By contrast, the method of alternative forms of down-arrows tells us the reverse: it tell us immediately whether a proposition is supporting or challenging what it points at, but the role of the support or challenge in the overall argument has to be worked out in relation to the chain of arrows back to the conclusion.

In order to resolve these difficulties (if they are perceived as such), one might use both methods, that is, two types of arrow (regular and dashed) and two directions (downward and upward). A regular arrow might mean (when pointing at a number) "is intended as sufficient for accepting <the proposition pointed at $>$ as true" and a dashed arrow might mean "is intended as sufficient not to accept $<$ the proposition pointed at $>$ as true" or (when pointing at an arrow) "is intended as sufficient for believing that $<$ the inference pointed at $>$ is not cogent". Using this double convention, (8)'s objection to (7) and (9)'s rebuttal to (8) are given dashed arrows (or any other different kind of arrow), while a proposition expressing support for the truth of (8) would receive a regular arrow, though point up towards (8). The diagram, along with a further proposition (10) would look as follows: 


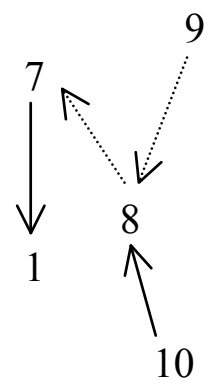

One can easily see in this diagram that, from the type of arrow used, (10) offers support for (8), which is an objection to the truth of (7), and, from the fact that they both are up-arrows, that in the overall argument they both serve, indirectly, to challenge (1). Similarly, (9) is an objection to the truth of (8) and is, indirectly, support for (1).

\section{Summary}

In sum, the proposals are these:

1. Each basic reasoning structure should be represented by a single arrow. Independent-premise arguments which are clearly signed as involving added support can employ a single arrow and any indication of added support other than whatever is used for dependent premises (typically the plussign). Arguments involving an unstructured pile of reasons are to be diagrammed with a split-tailed arrow.

Indeterminate Structure

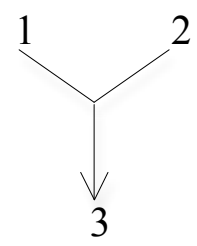

It is (deliberately) not clear from this diagram whether we have two arguments, or added support, or any other structure. Examples include most non-academic reasoning, including editorials.

2. Objections can point at the number representing a premise whose truth is being challenged or at the arrow of the inference of the arrow being challenged, and in diagrams of arguments of indeterminate structure, either at the arrow's 
main shaft or at a specific tail. (Counter-arguments can point at the number representing the conclusion.)

3. An objection might be recognized as such by the different type of arrow employed and/or by the difference in direction between the arrow representing either the arrow it points at (if challenging an inference) or to the arrow out of the number pointed at (if challenging the truth of a proposition) or the arrow into the number for the conclusion pointed at (if a direct argument that the conclusion is false).

4. An arrow representing a rebuttal to an objection might go in the opposite direction to the arrow representing the objection, and an arrow representing support for an objection in the same direction as the objection. Regular and irregular arrows can also be used to indicate the impact of the proposition at which each arrow points.

Acknowledgements: Thanks are due to Bill Melanson and Ryan Nichols, audience members at an AAPT session and two anonymous referees for this journal.

\section{References}

Araucaria http://araucaria.computing.dundee.ac.uk/doku.php

Austhink.com http://austhink.com/reason/tutorials/

Epstein, R.L. (2002). Critical Thinking, $3^{\text {rd }}$ ed. Belmont, CA: Wadsworth.

Kelley, D. (1988). The Art of Reasoning. New York: W.W. Norton.

Johnson, R.H. \& J.A. Blair. (1994). Logical Self-Defense, $1^{\text {st }}$ U.S. ed. New York: McGraw-Hill.

Moore, B.N. \& R. Parker, (2001). Critical Thinking, $6^{\text {th }}$ ed. Mountain View, CA: Mayfield.

Snoeck Henkemans, A.F. (1992). Analysing Complex Argumentation. Amsterdam: Sicsat.

Thomas, S.N. (1986). Practical Reasoning in Natural Language, $3^{\text {rd }}$ ed. Englewood Cliffs, NJ: Prentice-Hall.

Toulmin, S.E. (1984). The Uses of Argument. Cambridge: Cambridge University Press. (First issued in 1958.)

Walton, D. (2006). Fundamentals of Critical Argumentation. Cambridge: Cambridge University Press.

Yanal, R.J. (1991). "Dependent and Independent Reasons.” Informal Logic 13.3: 137-144.

Yoshimi, J. (2004). "Mapping the Structure of Debate." Informal Logic 24.1: 1-21. 